\title{
On the activation energy of the formic acid oxidation reaction on platinum electrodes.
}

\author{
Juan V. Perales-Rondón, Enrique Herrero* and Juan M. Feliu. \\ Departamento de Química-Física, Instituto de Electroquímica, Universidad de Alicante \\ Apartado 99, E-03080, Alicante, Spain.E-mail: herrero@ua.es
}

\begin{abstract}
.
A temperature dependent study on the formic acid oxidation reaction has been carried out in order to determine the activation energy of this reaction on different platinum single crystal electrodes, namely $\operatorname{Pt}(100), \operatorname{Pt}(111), \operatorname{Pt}(554)$ and $\operatorname{Pt}(544)$ surfaces. The chronoamperometric transients obtained with pulsed voltammetry have been analyzed to determine the current densities through the active intermediate and the $\mathrm{CO}$ formation rate. From the temperature dependency of those parameters, the activation energy for the direct reaction and the CO formation step have been calculated. For the active intermediate path, the activation energy are in the range of 50-60 kJ/mol. On the other hand, a large dependence on the electrode potential is found for the activation energy of the $\mathrm{CO}$ formation reaction on the $\mathrm{Pt}(100)$ electrode, and the activation values for this process range between 20 and $100 \mathrm{~kJ} / \mathrm{mol}$. These results have been explained using a reaction mechanism in which the oxidation of formic acid requires the presence of a pre-adsorbed anion on the electrode surface.
\end{abstract}

Keywords: Platinum, formic acid oxidation, single crystal electrodes, fuel cell. 


\section{Introduction.}

The formic oxidation reaction has been extensively studied because its potential use as anode reaction in the fuel cell technology. Additionally, from all the possible carbon containing species studied as tentative fuels, it has the simplest oxidation mechanism [1-3]. In fact, its oxidation to $\mathrm{CO}_{2}$ only requires the cleavage of two bonds: $\mathrm{O}-\mathrm{H}$ and $\mathrm{C}-\mathrm{H}$. The break of the $\mathrm{O}-\mathrm{H}$ bond is relatively simple, since the $\mathrm{OH}$ group is involved in the acid/base equilibria of the molecule. For the second one, a surface with affinity for hydrogen and dehydration reactions is required. For that reason, the platinum and palladium electrodes show the highest activity.

In spite of the apparent simplicity of the reaction, additional complications in the oxidation mechanism appear on platinum surfaces. On these electrodes, CO formation at low potentials is observed [1]. To produce adsorbed CO, the cleavage of two different bonds should take place: the $\mathrm{C}-\mathrm{H}$ and the $\mathrm{C}-\mathrm{OH}$ bonds. The formation of $\mathrm{CO}$ creates additional problems in the oxidation of the formic acid molecule. CO is strongly adsorbed on the Pt surface and its final oxidation to $\mathrm{CO}_{2}$ requires the transfer of an $\mathrm{OH}$ group. This latter step has a large overpotential. For this reason, $\mathrm{CO}$ is also called the poisoning intermediate, since it blocks the surface for the reaction to continue. On the other hand, the reaction route which gives directly $\mathrm{CO}_{2}$ is called the direct oxidation route. For this route, the nature of the intermediate is still subject of discussion [4-11].

In order to get insight in to the oxidation mechanism, extensive DFT calculations have been carried out using well defined surfaces [12-15]. In these studies, different intermediates and reaction schemes have been proposed. However, in order to validate those reaction mechanism, the theoretical data for the calculated activation energies should be compared with those obtained experimentally. In this manuscript the activation energies for the formic acid oxidation reaction are measured on single crystal electrodes using different electrolytes. 
The activation energies will be analyzed in view to the proposed mechanism, and compared with previous results, in order to obtain additional details on the oxidation mechanism. In this way, the measured values will be compared with those obtained from DFT calculations to validate the proposed mechanism.

\section{Experimental procedure and numerical treatment of data.}

\subsection{Experimental procedure}

Platinum single crystal electrodes were oriented, cut and polished from small single crystal beads (ca. $2.5 \mathrm{~mm}$ diameter) following the procedure described by Clavilier [16, 17]. The electrodes used in this study were the $\operatorname{Pt}(111)$ and $\operatorname{Pt}(100)$ low index planes and two stepped surfaces: $\operatorname{Pt}(544)$ and $\operatorname{Pt}(554)$. These surfaces have 9 atom-wide (111) terraces separated by monoatomic (100) and (110) steps, respectively. Before every use, the electrodes were cleaned by flame annealing, cooled down in $\mathrm{H}_{2} / \mathrm{Ar}$ and protected with water in equilibrium with this atmosphere. It is known that this procedure gives rise to surfaces whose atomic arrangement corresponds to the nominal one [18]. The voltammetric profile of the electrode in $0.5 \mathrm{M} \mathrm{H}_{2} \mathrm{SO}_{4}$ is always recorded to assure that the surface is well prepared and clean. The upper potential limit is always chosen so that oxide formation is avoided, to prevent surface disordering $[19,20]$.

Experiments were carried out in a classical two-compartment electrochemical cell deaerated by using Ar (N50, Air Liquide in all gases used), including a large platinum counter electrode and a reversible hydrogen (N50) electrode (RHE) as reference. For the temperature controlled experiments, the electrochemical cell was immersed in a water bath to control the temperature. Temperature dependent measurements were conducted in a range between 278 and $333 \mathrm{~K}$, using $5 \mathrm{~K}$ intervals. For the different experiments, the reference electrode was kept at room temperature ( $298 \mathrm{~K}$ ). To transform the direct potential reading to the RHE scale 
at $298 \mathrm{~K}$ values, the direct potential reading should be corrected for the thermodiffusion potential using the procedure explained in reference [21]. Solutions were prepared from sulfuric acid, perchloric acid, sodium perchlorate, formic acid (Merck suprapur in all cases) and ultrapure water from Elga.

The potential program for the pulse voltammetry which allowed obtaining the current transients was generated with an arbitrary function generator (Rigol, DG3061A) together with a potentiostat (eDAQ EA161) and a digital recorder (eDAQ, ED401). To avoid any interference of the diffusion of formic acid in the reaction rate, stationary conditions were attained by using a hanging meniscus rotating disk configuration at $900 \mathrm{rpm}$ (controlled by a Radiometer CTV 101). Full experimental details on the pulsed voltammetry can be found in reference [22]. In summary, the potential program consists in a series of steps between an upper potential ( $0.9 \mathrm{~V}$ for $\mathrm{T}<298 \mathrm{~K}$ and $0.85 \mathrm{~V}$ for $\mathrm{T} \geq 298 \mathrm{~K}$ ) to oxidized the accumulated $\mathrm{CO}$ on the surface and the sampling potential. The duration of the steps was always $1 \mathrm{~s}$.

\subsection{Numerical treatment of the transients.}

In a previous work, transients obtained in the pulsed voltammetry as those shown in figure 1 were simulated with a model which took into account direct formic acid oxidation and $\mathrm{CO}$ formation through the dehydration reaction [22]. For the oxidation through the active intermediate, it was supposed that the current density depended on the fraction of the surface not covered by $\mathrm{CO},\left(1-\theta_{\mathrm{CO}}\right)$, and the current that would have been obtained in absence of $\mathrm{CO}$, $j_{\theta=0}$.

$$
j=j_{\theta=0}\left(1-\theta_{\mathrm{CO}}\right)
$$

In turn, the CO coverage will depend on the on the dehydration step, for which the reaction rate was written as: 


$$
\frac{d \theta_{C O}}{d t}=k_{a d s}\left(1-\theta_{\mathrm{CO}}\right)^{p}
$$

where $k_{a d s}$ is the poison formation reaction rate and $p$ is the number of Pt sites required for the dehydration step to occur. The integration of equation (2), and substitution in equation (1) yielded:

$$
j=j_{\theta=0}\left(\frac{1}{1+k_{a d s} t(p-1)}\right)^{\frac{1}{p-1}}
$$

This model was used to obtain the values of $j_{\theta=0}, k_{a d s}$ and $p$ from the fits of this equation to the experimental transients. The fits were very good at room temperature, which supported the validity of the model $[22,23]$. In all cases, $p$ was close to two, which implies that the dehydrogenation reaction requires two contiguous free platinum sites to proceed. However, when this equation is for $T>298 \mathrm{~K}$ for the transients obtained in this work, the model deviates from experimental transients (figure 1, dotted line). In the model represented by equation (3), possible contributions to the total current from the adsorbed CO oxidation process according to the reaction

$$
\mathrm{CO}+\mathrm{H}_{2} \mathrm{O} \rightarrow \mathrm{CO}_{2}+2 \mathrm{H}^{+}+2 \mathrm{e}
$$

were not taken into account, because it was experimentally verified that, the CO oxidation rate was negligible at the potentials where $\mathrm{CO}$ was formed [22, 23]. In the worst case scenario, the $\mathrm{Pt}(100)$ electrode, $\mathrm{CO}$ formation takes place at $E<0.50 \mathrm{~V}$ and for those potentials $\mathrm{CO}$ oxidation is negligible at room temperature. However, the temperature increase has two major effects: to accelerate $\mathrm{CO}$ oxidation process and also to increase the potential window where CO formation occurs. For this reason, the deviations from previous model are due to the contribution of the $\mathrm{CO}$ oxidation process to the measured current and, thus, such contribution should be taken into account for the transients at $T>298 \mathrm{~K}$. Equation (2) should 
be then modified incorporating the possibility of change of CO coverage due to its oxidation according to the proposed mean field Langmuir-Hinselwood mechanism [3, 24, 25] as:

$$
\frac{d \theta_{\mathrm{CO}}}{d t}=k_{\text {ads }}\left(1-\theta_{\mathrm{CO}}\right)^{p}-k_{o x} \theta_{\mathrm{CO}}\left(1-\theta_{\mathrm{CO}}\right)
$$

where $k_{o x}$ is the rate constant for the CO oxidation process. In the same way, the equation for the current density should incorporate the contributions to the measured current of the CO oxidation reaction as:

$$
j=j_{\theta=0}\left(1-\theta_{\mathrm{CO}}\right)+q k_{o x} \theta_{\mathrm{CO}}\left(1-\theta_{\mathrm{CO}}\right)
$$

where $q$ is the charge involved to the oxidation of a monolayer of $\mathrm{CO}\left(320 \mu \mathrm{C} \mathrm{cm}^{-2}\right.$ for the $\operatorname{Pt}(100)$ electrode). In order to solve equation (5) for $\theta_{\mathrm{CO}}, p$ was fixed to 2 , because this was the value obtained experimentally [22]. Assuming that $k_{a d s}>>k_{o x}, \theta_{\mathrm{CO}}$ is then:

$$
\theta_{\mathrm{CO}}=k_{a d s} \frac{\exp \left(k_{o x} t\right)-1}{k_{o x} \exp \left(k_{o x} t\right)+k_{a d s} \exp \left(k_{o x} t\right)-k_{a d s}}
$$

Substituting into equation (6) gives:

$$
j=\frac{k_{o x}\left[\left(1-\exp \left(-k_{o x} t\right)\right)\left(j_{\theta=0} k_{a d s}+q k_{a d s} k_{o x}\right)+j_{\theta=0} k_{o x}\right]}{\left[k_{o x}+k_{a d s}\left(1-\exp \left(-k_{o x} t\right)\right)\right]^{2}}
$$

Of course, when $k_{o x}$ tends to 0 , equation (8) transforms into equation (3) with $p=2$. As can be seen, the model, which also contains 3 adjustable parameters, as the previous one, is able to perfectly fit the experimental transients (figure 1, dashed line). 


\section{Results and discussion.}

\subsection{Temperature effects on the behavior of the electrodes in the pure supporting}

electrolyte.

It has been shown that the formic acid oxidation reaction is affected by the anion adsorption process. In fact, it has been proposed that anion adsorption is required for the oxidation to proceed [26]. For the $\mathrm{Pt}(111)$ electrode, the proposed model suggests that the formic acid oxidation reaction requires the presence of an anion adsorbed on the surface and a contiguous free site. On this free site, formate, the proposed reactive species [8], can interact with the surface in a configuration which favors the C-H bond cleavage [27]. DFT calculations indicate that the activation energy for the cleavage of the $\mathrm{C}-\mathrm{H}$ bond is low when the this bond is directed to the surface [14], and thus it can be proposed that the formate species should have the $\mathrm{C}-\mathrm{H}$ bond directed to the surface. The adsorbed anion that facilitates the adsorption of formate in the $\mathrm{C}-\mathrm{H}$ down configuration can be either a formate species bonded to the surface through one or two oxygen atoms or any other anion such as acetate or sulfate [26]. Thus, the adsorption of the anion and how this process is influenced by the temperature is an important parameter that can affect the measured activation energy.

Figure 2 shows the voltammetric profiles of the electrodes used in this work at different temperatures. For the $\operatorname{Pt}(111)$ electrode, as the temperature increases, sulfate adsorption shifts slightly towards more positive potentials [28, 29]. All the signals related to sulfate adsorption move slightly to more positive potentials, but the effect is clearer when the position of the spike at ca. $0.45 \mathrm{~V}$ is compared for the different temperatures. This spike marks the position of the disorder-order transition of the sulfate layer [30], and this transition, which takes place at a constant coverage [31], moves towards positive potentials. This fact clearly indicates that the adsorption of anions is disfavored as the temperature increases. The second feature, the small bump at 0.6-0.7 is related the completion of the sulfate layer [32]. In 
this case, this process is more reversible with the temperature, as the peak separation between positive and negative scan direction diminishes, but there is no significant peak shift.

The situation for the $\mathrm{Pt}(100)$ electrode is different. For this electrode, there is no clear separation between hydrogen and anion adsorption processes. The peak at ca. $0.37 \mathrm{~V}$ marks the point where hydrogen adsorbed atoms are being replaced by adsorbed sulfate [33]. As can be seen, this peak shifts towards negative potential values, but is less sharp [29]. This fact indicates that the onset for the adsorption process takes place at lower potentials. However, the sharpness of the peak indicates how narrow the window is for the replacement of the adsorbed hydrogen atoms by sulfate. This would suggest that the adsorption of the sulfate anion starts at lower potentials but the completion of the full adlayer shifts towards higher potential values as the temperature increases. For the other two surfaces, there is no noticeable change of the voltammetric profile as the temperature increases, which indicates no major changes in anion adsorption with temperature.

The differences in the anion adsorption between the low index and stepped surfaces stress the significant effects of the long range order in the adsorption of anion for large planar surfaces. For the $\operatorname{Pt}(100)$ and $\operatorname{Pt}(111)$ electrodes, achieving a long range order in the sulfate adlayer is the key factor for the completion of the anion layer [32]. As aforementioned, the small bump between 0.6-0.7 V for the Pt(111) electrode is related to the completion of the adalyer. This process becomes more reversible as the temperature increases, revealing a strong effect of the temperature in this process. On the other hand, when the terrace size diminishes, the long range order effects are less important in the adsorption processes, because it is interrupted by the presence of steps, and thus, the adlayer formation is depends only in the individual adsorption energies of the different sites, which are less affected by the temperature. 


\subsection{Effects of the temperature and anion adsorption on the formic acid oxidation}

reaction.

The voltammetric profiles for the formic acid oxidation reaction at $298 \mathrm{~K}$ in perchloric and sulfuric acid solutions are shown in figure 3. In all cases, there is an hysteresis between positive and negative scan directions, due to the formation of $\mathrm{CO}$ through the dehydration reaction at low potentials $[22,23]$. Thus, currents in the positive scan direction are affected by the presence of adsorbed CO, which blocks the surface for the direct oxidation reaction. At high potentials $(E>0.75 \mathrm{~V})$, $\mathrm{CO}$ is readily oxidized and the surface recovers its activity. In the negative scan direction, the surface is not blocked by $\mathrm{CO}$ for $\mathrm{E}>0.5 \mathrm{~V}$ and for that reason, currents are much higher. The relative difference between the currents in positive and negative san directions is related to the kinetics of $\mathrm{CO}$ formation at low potentials. For the Pt(100), since the CO formation rate is the highest of all basal planes, currents are almost zero in the positive scan direction. A similar situation is observed for the $\mathrm{Pt}(554)$, because the $\mathrm{CO}$ formation rate on the (110) monoatomic step is also high [23]. On the other hand, for the Pt(111), the hysteresis is very small, since CO formation is related to defects. An intermediate situation is observed for the $\mathrm{Pt}(544)$ electrode, where the activity of the (100) steps for the CO formation step is not very high [23].

As expected, maximum currents for formic acid oxidation in perchloric acid are higher than those measured in sulfuric. However, the difference for the $\operatorname{Pt}(100)$ and $\operatorname{Pt}(111)$ are very small. Moreover, for the $\operatorname{Pt}(111)$ electrode, currents at $E<0.4 \mathrm{~V}$ are larger in sulfuric acid. These facts reveal that adsorbed sulfate is not inhibiting formic acid oxidation on these two surfaces. On the other hand, for the stepped surfaces, the differences are somehow larger. Since the behavior of the (111) plane for the oxidation is the same in perchloric and sulfuric acid solutions, as figure 3 demonstrates, the larger differences should be then associated to the different activity of the steps. For these surfaces, adsorbed sulfate on the steps should have an 
inhibiting behavior, whereas the adsorption on the (100) or (111) planes is neutral or even facilitates the formic acid oxidation reaction [27].

The effect of the temperature on the formic acid oxidation reaction is shown in figure 4. For simplicity, only negative scan directions are shown. The activity is mainly related to the direct oxidation of formic acid for this scan direction, since the surface is free from $\mathrm{CO}$ at the beginning of the scan $(E=0.90 \mathrm{~V})$. For the $\operatorname{Pt}(100)$ electrode, currents at $E<0.4 \mathrm{~V}$ are almost independent of the temperature and above $0.4 \mathrm{~V}$, a clear increase is observed. A priori, this constant value would suggest that the activation energy is zero for $E<0.4 \mathrm{~V}$, since the dependence of the current with temperature is negligible. However, the decay in the current in the negative scan direction after the peak is mainly related to the formation of $\mathrm{CO}$ which blocks the surface [22], and, thus, the activation energy for the direct path should be higher. As will be shown later, the rate for $\mathrm{CO}$ formation in this region also increases with the temperature, and the observed behavior is just a consequence of two opposing factors: the increasing rate of the direct reaction and of the dehydration step to yield $\mathrm{CO}$, which results in currents that are almost independent of the temperature. For the other surfaces, a clear increase in current with the temperature is observed in all cases.

\subsection{Activation energy for the reaction through the active intermediate.}

The different transients measured with the pulsed voltammetry were analyzed using equation (8) and the values of $j_{\theta=0}$ and $k_{a d s}$ from the different electrodes at different potentials and temperatures were calculated. Figure 5 shows the values of $j_{\theta=0}$ for the different electrodes used in this study. The voltammetric profile in the negative scan direction at $45{ }^{\circ} \mathrm{C}$ is also shown for comparison. For the $\operatorname{Pt}(111), \operatorname{Pt}(544)$ and $\operatorname{Pt}(554)$ electrodes, values of $j_{\theta=0}$ and measured current densities in negative scan direction of the voltammogram are almost identical for $E<0.3 \mathrm{~V}$, which indicates that poison is not formed in this potential region, as 
was observed previously for room temperature [23]. Also, oxidation transients are almost horizontal and the $k_{a d s}$ values obtained from the fittings are negligible. In fact, for the Pt(544) and $\mathrm{Pt}(554)$, CO is formed in the around the peaks associated to the adsorption of hydrogen on the steps in the potential region around 0.26 and $0.13 \mathrm{~V}$, respectively (see figure 1) [23]. For the $\operatorname{Pt}(100)$ electrode, there is a significant difference between $j_{\theta=0}$ and the measured current for $E<0.5 \mathrm{~V}$. The lower currents in the voltammetry are clearly related to the blocking of the surface by CO through the dehydration reaction, which is formed at $E<0.5 \mathrm{~V}$ (see figure 8).

Activation energies for the direct oxidation were obtained from Arrhenius plots $\left(\log \left(j_{\theta=0}\right)\right.$ vs. $\left.1 / T\right)$. In all cases, these plots are linear. It should be stressed that the measured activation energy is an apparent activation energy $\left(E a_{a p p}^{\neq}\right)$and contains terms that are not directly related to the activation energy of the different steps. It has been proposed that the oxidation through the active intermediate follows the rate equation [27]:

$$
j_{\theta=0}=F k^{o} \exp \left(\frac{\alpha F\left(E-E^{o}\right)}{R T}\right) \theta_{\mathrm{A}}\left(1-\theta_{\mathrm{A}}-\theta_{\mathrm{OH}}\right)\left[\mathrm{HCOO}^{-}\right]
$$

where $k^{o}$ is the standard rate constant, $E^{o}$ the standard potential for the oxidation of formic acid, $\alpha$ is the symmetry factor of the reaction, $\theta_{\mathrm{A}}$ and $\theta_{\mathrm{OH}}$ are the specific anion and $\mathrm{OH}$ coverages and the rest of symbols have the usual meaning. This rate equation corresponds to a mechanism whose rds is a step in which a formate anion interacts with the surface (in a free site) in the C-H down configuration close to an adsorbed anion (formate or sulfate), as has been proposed by the $\mathrm{pH}$ dependence of the oxidation rate of formic aicd [8]. This rds, in which the $\mathrm{C}-\mathrm{H}$ bond is cleaved and an electron is transferred, corresponds to the second electron transfer.

$$
\mathrm{Pt}-\mathrm{A}+\mathrm{Pt}-\mathrm{HCOO}_{\mathrm{C}-\mathrm{H} \text { down }}^{-} \rightarrow \mathrm{Pt}-\mathrm{A}+\mathrm{CO}_{2}+\mathrm{Pt}-\mathrm{H}+\mathrm{e}^{-}
$$

The activation energy of the reaction is defined as: 


$$
E a^{\neq}=-R\left(\frac{d \ln \left(k^{o}\right)}{d \frac{1}{T}}\right)_{E, \theta,[\text { HCOO }]}
$$

whereas the actually measured activation energy corresponds to the derivative $\ln \left(j_{\theta=0}\right)$ vs. $1 / \mathrm{T}$, according to:

$$
E a_{a p p}^{\neq}=-R\left(\frac{d \ln \left(j_{\theta=0}\right)}{d \frac{1}{T}}\right)_{E}=E a^{\neq}-R\left(\frac{d \ln \left(\theta_{\mathrm{A}}\right)}{d \frac{1}{T}}+\frac{d \ln \left(1-\theta_{\mathrm{A}}-\theta_{\mathrm{OH}}\right)}{d \frac{1}{T}}\right)_{E}-\alpha F\left(E-E^{0}\right)
$$

Thus, it contains terms that are related to the effect of the temperature on the anion and $\mathrm{OH}$ coverage and the electrode potential, which can affect the measured activation energy and modify its value. If the terms related to the coverages were negligible (that is, they were independent of the temperature), the apparent activation energy for the oxidation reaction should diminish with the electrode potential according to:

$$
E a_{a p p}^{\neq}=-R\left(\frac{d \ln \left(j_{\theta=0}\right)}{d \frac{1}{T}}\right)_{E}=E a^{\neq}-\alpha F\left(E-E^{0}\right)
$$

If this is not true, that is, if the terms related to the coverages are temperature dependent, deviations from this slope must be observed. The potential regions where those terms can have a larger impact in the measured values are at the initial and final stages of the adsorption of the anions and OH. In the initial stages, the term $\frac{d \ln \left(\theta_{\mathrm{A}}\right)}{d \frac{1}{T}}$ should have the larger effect, since, at these potentials, temperature changes can have a relative large effect on the anion coverage. On the other hand, the $\frac{d \ln \left(1-\theta_{\mathrm{A}}-\theta_{\mathrm{OH}}\right)}{d \frac{1}{T}}$ term should have large values at the final stages of the adsorption process. 
Figure 6 and 7 shows the apparent activation energies obtained in perchloric and sulfuric solutions for the different electrodes. The dashed line shows the expected behavior for the apparent activation energy from equation (13) with $\alpha=1,5$ (the typical value for a mechanism in which the second electron transfer is the r.d.s) and an arbitrary value of $E a^{\neq}$. As can be seen, experimental values deviates clearly from the expected slope, which can be a consequence of the impact of the terms related to the anion and $\mathrm{OH}$ coverages in the formic acid oxidation process. The behavior of the $\mathrm{Pt}(111)$ electrode and the two vicinal surfaces $\operatorname{Pt}(554)$ and $\operatorname{Pt}(544)$ will be discussed first. In perchloric acid solutions, values are almost constant for these electrodes between 0.35 and $0.65 \mathrm{~V}$. Apparent activation energies are ca. 47, 50 and $52 \mathrm{~kJ} \mathrm{~mol}^{-1}$ for the $\operatorname{Pt}(111), \operatorname{Pt}(544)$ and $\operatorname{Pt}(554)$ in this region. First of all, the difference between these surfaces should be related to the activation energy on the (100) and (110) step sites. These values suggests that the activation energy on those sites is higher than in the (111) plane. Second, the almost constant value suggests that the terms related to the anion coverage either are very important, that is, significant changes in the anion coverages are observed with the temperature, or that additional factors, which have not been take into account, play a role in the mechanism. From $E>0.65 \mathrm{~V}$, the activation energy diminishes, with a behavior similar to that of equation (13). In this region, the formate coverage has reached to a maximum value, and probably, no change in the coverage is observed with the temperature.

In sulfuric acid solution, values for the $\operatorname{Pt}(554)$ and $\operatorname{Pt}(544)$ are very similar to those obtained in perchloric acid solution (figure 7). This fact indicates that adsorbed sulfate or formate, species that are required for the oxidation of formic acid, have very similar behavior, and supports the proposed mechanism [27]. There are only small changes at the onset potential, probably due to small difference on how temperature affects the initial stages of this process. Larger differences are observed on the Pt(111) electrode, for which, a diminution of the activation energy is observed around $0.6 \mathrm{~V}$ in sulfuric acid solutions. The diminution 
should be then related to the changes in the sulfate coverage, since, in this region the sulfate layer rearranges to reach the maximum coverage [21].

For the $\mathrm{Pt}(100)$ electrode in perchloric acid solution, there is an initial diminution, as predicted by equation (13), followed by a fast increase with the potential, reaching an almost constant value of $60 \mathrm{~kJ} \mathrm{~mol}^{-1}$ for $E>0.5 \mathrm{~V}$. The increase is related to the adsorption of anions, and it is more evident in sulfuric acid solutions. As temperature effects in the blank voltammetric profiles demonstrate, the anion coverages diminishes for $E>0.36 \mathrm{~V}$, which leads to an increase of the apparent activation energy due to the contribution of the $\frac{d \ln \left(1-\theta_{\mathrm{A}}-\theta_{\mathrm{OH}}\right)}{d \frac{1}{T}}$ term. In sulfuric acid solutions, the shape of the apparent activation energy curve in sulfuric acid solutions is similar to that measured in perchloric acid, but the local maxima and minima are more pronounced, related to a large effect of the temperature on the adsorption of sulfate over formate. As before, for this medium and $E>0.5 \mathrm{~V}$, the observed behavior follows equation (13), because sulfate coverage has reached to a maximum value, and the terms related to the coverage are temperature independent in this region.

In summary, the apparent activation energies for the different surfaces are in the range of ca. 50-60 kJ mol ${ }^{-1}$, once the effects of the anion adsorption in the measured activation energies are subtracted. This results contrasts with the significant larger currents measured for the Pt(100) electrode. Thus, the larger currents and, therefore, the larger kinetic constant for this latter electrode should be then linked to a higher pre-exponential factor in kinetic constant. Since the pre-exponential factor is related to the probability of reaching the configuration of the activated complex, this could imply that the initial configuration giving rise to the activated complex is more probable on the $\mathrm{Pt}(100)$ surface. This would suggests that, from the different adsorption modes of formate, the one having the configuration required for the reaction to proceed is more likely on the $\operatorname{Pt}(100)$ surface than in the rest. 


\subsection{Activation energy of the dehydrogenation process of formic acid to yield adsorbed} CO.

As aforementioned, for the $\operatorname{Pt}(111), \operatorname{Pt}(554)$ and $\operatorname{Pt}(544), k_{a d s}$ values were negligible for the region where non-zero oxidation transients for the formic acid oxidation reaction are obtained $(E>0.3 \mathrm{~V})$. However, for the $\mathrm{Pt}(100)$, it was possible to determine the values of $k_{a d s}$ from the transients in this region. Figure 8 shows the measured values for $k_{a d s}$ on this electrode at three representative temperatures. As can be seen, CO formation takes place in a potential region between 0.3 and $0.5 \mathrm{~V}$ with a maximum rate located close to the potential of zero total charge of the electrode [22]. This narrow regions expands as the temperature increase. The onset potential for the reaction shifts slightly towards lower potential values, whereas the upper limit for the reaction shifts markedly to higher values as the temperature increases.

Apparent activation energies for this reaction were calculated as before and the results are plotted in figure 9. In this figure, the apparent activation energy for the direct oxidation measured in the same experiment is also shown for comparison. As can be seen, the curve for the dehydration reaction presents the same features, with local minima and maxima located in the same potential, at 0.35 and $0.48 \mathrm{~V}$. The only differences are the values and the intensities of the maxima and minima. If we accept that the deviations from the expected value are related to the anion coverage terms in the kinetic equation, the kinetic equation should be similar but with a higher order for the terms related to the anions in the kinetic equation. In fact, in the fittings of the transients, it was assumed that the reaction requires two free sites to proceed [22], and thus, it can be written that the rate for the reaction is:

$$
k_{\text {ads }}=k_{\text {ads }}^{o} \theta_{\mathrm{A}}\left(1-\theta_{\mathrm{A}}-\theta_{\mathrm{OH}}\right)^{2}\left[\mathrm{HCOO}^{-}\right]
$$


where $k_{a d s}^{o}$ is the standard rate constant for the process. This equation qualitatively justifies the larger change in the activation energy due to the changes in the anion coverage due to the exponential factor of the (1- $\left.\theta_{\mathrm{A}}-\theta_{\mathrm{OH}}\right)$ term and also the observed shape of the curves of $k_{a d s} \mathrm{vs}$. potential, which should have a maximum located at $\theta_{\mathrm{A}}=1 / 3$ and an onset potential which coincides with the onset potential of the anion and a final potential in the region where the anion adlayer is completed (or the anion has been replaced by adsorbed $\mathrm{OH}$ ). It is not possible to predict the exact shape of the curves, since isotherms for the anion adsorption on the $\operatorname{Pt}(100)$ are not currently available, but the general shape agrees with the model. Incidentally, the changes with the temperature are also in agreement with the observed behavior of the anion adsorption on the $\operatorname{Pt}(100)$ electrode as the temperature increases. As aforementioned, the onset for anion adsorption is displaced to lower values as the temperature increases, and the same behavior is observed for $k_{a d s}$. Also, the completion of the anion adlayer requires higher applied potentials, and thus, the window where CO formation takes place expands towards positive values.

\section{Conclusions.}

In this manuscript, the apparent activation energies for the formic acid oxidation reaction on different platinum single crystal electrodes have been calculated, both for the direct and poisoning routes. It has been shown that the potential dependent behavior of the activation energies for the direct route is in agreement with a mechanism in which the adsorbed anions present on the surface play an active role in the oxidation mechanism. It has been proposed that these anions (sulfate or formate itself) facilitates the adsorption of the formic acid molecule in a configuration in which the $\mathrm{C}-\mathrm{H}$ bond is directed towards the surface. Thus, the kinetic equation for the oxidation currents through the active intermediate contains the potential dependence, a term related to the pre-adsorbed anion coverage and 
another corresponding to a free site, where the incoming formate species should adsorb in the $\mathrm{C}-\mathrm{H}$ down configuration. In this configuration, results from the literature show that the activation energy is low. For the poisoning route, the adsorbed anions are also important. However, two free sites are required for the anion to adsorb. It is important to stress that the complete understanding of an oxidation mechanism can only be achieved if experimental results using different techniques are combined with theoretical calculations.

\section{Acknowledgments.}

This work has been financially supported by the MICINN (Spain) (project 2013-44083-P FEDER) and Generalitat Valenciana (project PROMETEOII/2014/013, -FEDER).

\section{References}

[1] R. Parsons, T. Vandernoot, J. Electroanal. Chem., 257 (1988) 9.

[2] J.M. Feliu, E. Herrero, in: W. Vielstich, H. Gasteiger, A. Lamm (Eds.) Handbook of Fuel Cells - Fundamentals, Technology and Applications, vol. 2, John Wiley \& Sons, Ltd., Chichester, 2003.

[3] M.T.M. Koper, S.C.S. Lai, E. Herrero, in: M.T.M. Koper (Ed.) Fuel Cell Catalysis, A Surface Science Approach, John Wiley \& Sons, Inc, Hoboken, NJ, 2009, pp. 159.

[4] Y.X. Chen, M. Heinen, Z. Jusys, R.J. Behm, Langmuir, 22 (2006) 10399.

[5] G. Samjeske, M. Osawa, Angew. Chem. Int. Edit., 44 (2005) 5694.

[6] G. Samjeske, A. Miki, S. Ye, M. Osawa, J. Phys. Chem. B, 110 (2006) 16559.

[7] M. Osawa, K. Komatsu, G. Samjeske, T. Uchida, T. Ikeshoji, A. Cuesta, C. Gutierrez, Angew. Chem. Int. Edit., 50 (2011) 1159.

[8] J. Joo, T. Uchida, A. Cuesta, M.T.M. Koper, M. Osawa, J. Am. Chem. Soc., 135 (2013) 9991.

[9] Y.X. Chen, M. Heinen, Z. Jusys, R.B. Behm, Angew. Chem. Int. Edit., 45 (2006) 981.

[10] Y.X. Chen, M. Heinen, Z. Jusys, R.J. Behm, ChemPhysChem, 8 (2007) 380.

[11] S. Brimaud, J. Solla-Gullon, I. Weber, J.M. Feliu, R.J. Behm, ChemElectroChem, 1 (2014) 1075.

[12] M. Neurock, M. Janik, A. Wieckowski, Faraday Discuss., 140 (2009) 363.

[13] W. Gao, J.A. Keith, J. Anton, T. Jacob, J. Am. Chem. Soc., 132 (2010) 18377.

[14] H.-F. Wang, Z.-P. Liu, J. Phys. Chem. C, 113 (2009) 17502.

[15] J.V. Perales-Rondon, A. Ferre-Vilaplana, J.M. Feliu, E. Herrero, J. Am. Chem. Soc., 136 (2014) 13110.

[16] J. Clavilier, D. Armand, S.G. Sun, M. Petit, J. Electroanal. Chem., 205 (1986) 267. 
[17] C. Korzeniewski, V. Climent, J.M. Feliu, in: A.J. Bard, C. Zoski (Eds.) Electroanalytical Chemistry: A Series of Advances, vol. 24, CRC Press, Boca Raton, 2012, pp. 75.

[18] E. Herrero, J.M. Orts, A. Aldaz, J.M. Feliu, Surf. Sci., 440 (1999) 259.

[19] K. Itaya, S. Sugawara, K. Sashikata, N. Furuya, J. Vac. Sci. Technol. A, 8 (1990) 515.

[20] A.M. Gomez-Marin, J.M. Feliu, Electrochim. Acta, 82 (2012) 558.

[21] N. Garcia-Araez, V. Climent, J. Feliu, in: C.G. Vayenas (Ed.) Mod Asp Electrochem, vol. 51, Springer New York, 2011, Ch. 1, pp. 1.

[22] V. Grozovski, V. Climent, E. Herrero, J.M. Feliu, ChemPhysChem, 10 (2009) 1922.

[23] V. Grozovski, V. Climent, E. Herrero, J.M. Feliu, Phys. Chem. Chem. Phys., 12 (2010) 8822.

[24] M. Bergelin, E. Herrero, J.M. Feliu, M. Wasberg, J. Electroanal. Chem., 467 (1999) 74.

[25] N.P. Lebedeva, M.T.M. Koper, E. Herrero, J.M. Feliu, R.A. van Santen, J. Electroanal. Chem., 487 (2000) 37.

[26] V. Grozovski, F.J. Vidal-Iglesias, E. Herrero, J.M. Feliu, ChemPhysChem, 12 (2011) 1641.

[27] J.V. Perales-Rondon, E. Herrero, J.M. Feliu, Electrochim. Acta, 140 (2014) 511.

[28] S. Blais, G. Jerkiewicz, E. Herrero, J.M. Feliu, Langmuir, 17 (2001) 3030.

[29] E. Herrero, B. Alvarez, J.M. Feliu, S. Blais, Z. Radovic-Hrapovic, G. Jerkiewicz, J. Electroanal. Chem., 567 (2004) 139.

[30] A.M. Funtikov, U. Linke, U. Stimming, R. Vogel, Surf. Sci., 324 (1995) L343.

[31] E. Herrero, J. Mostany, J.M. Feliu, J. Lipkowski, J. Electroanal. Chem., 534 (2002) 79.

[32] N. Garcia-Araez, V. Climent, P. Rodríguez, J.M. Feliu, Electrochim. Acta, 53 (2008) 6793.

[33] J. Clavilier, J.M. Orts, R. Gómez, J.M. Feliu, A. Aldaz, in: B.E. Conway, G. Jerkiewicz (Eds.), vol. 94-21, The Electrochemical Society, INC., Pennington, NJ, 1994, pp. 167. 
Figures.

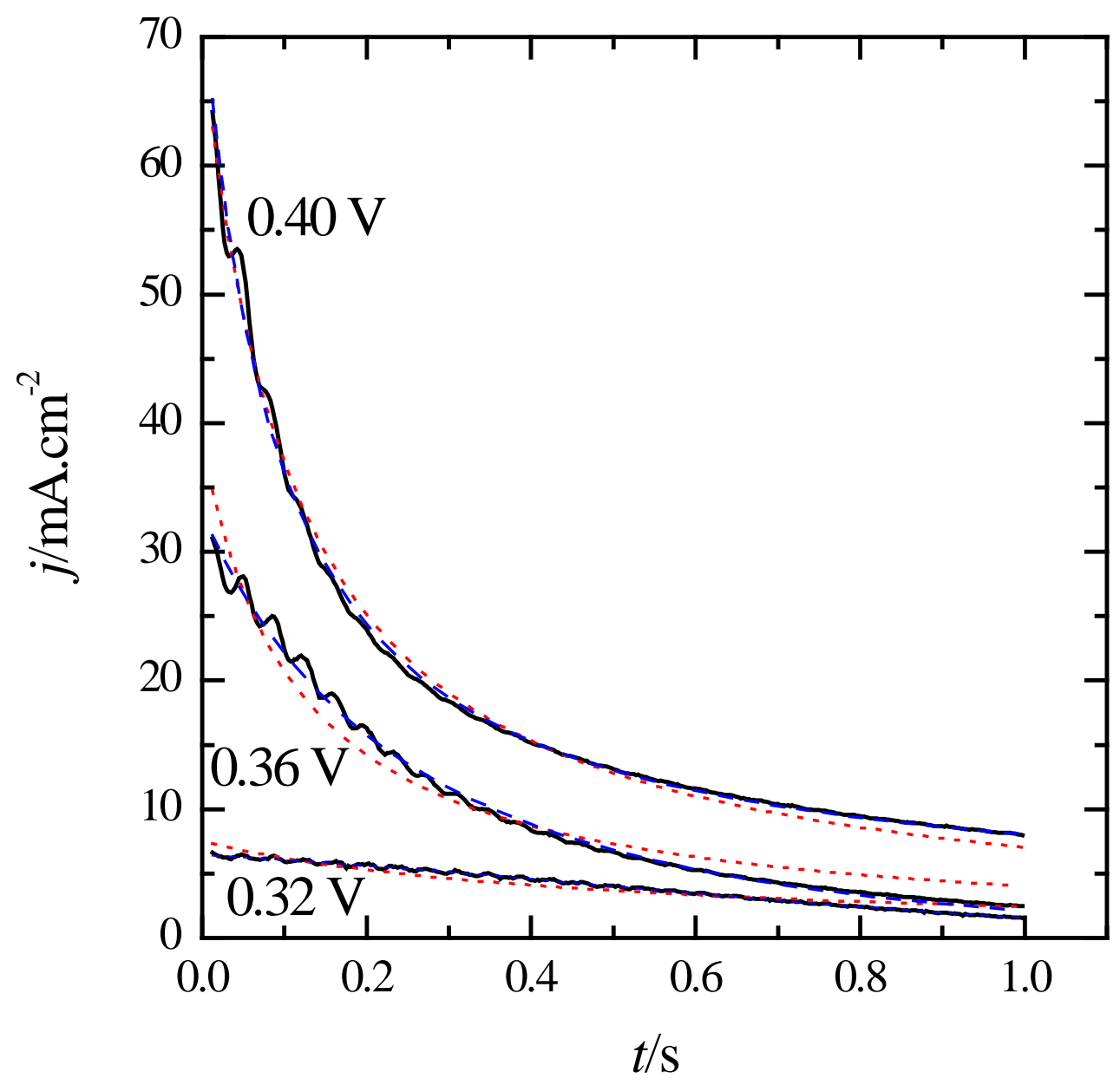

Figure 1. Transient currents measured during the pulsed voltammetry at different potentials on the $\operatorname{Pt}(100)$ electrode at $40{ }^{\circ} \mathrm{C}$ in $0.5 \mathrm{H}_{2} \mathrm{SO}_{4}+0.1 \mathrm{M} \mathrm{HCOOH}$ and fittings according to equation 3 (dotted line) and equation 8 (dashed line). 


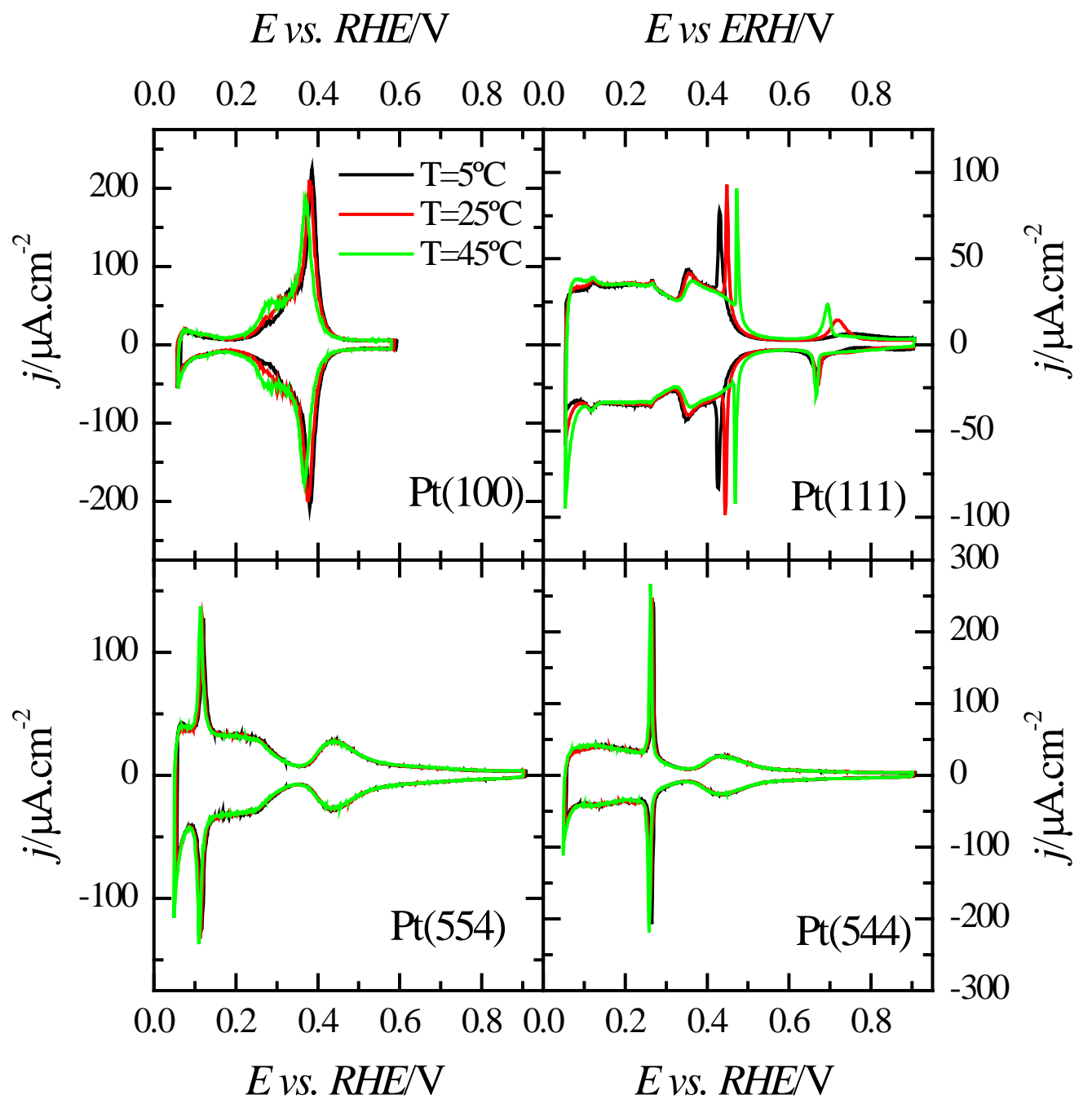

Figure 2. Effect of the temperature in the voltammetric profiles of the electrodes in $0.5 \mathrm{M}$ $\mathrm{H}_{2} \mathrm{SO}_{4}$. Scan rate: $50 \mathrm{mV} \mathrm{s}^{-1}$. 


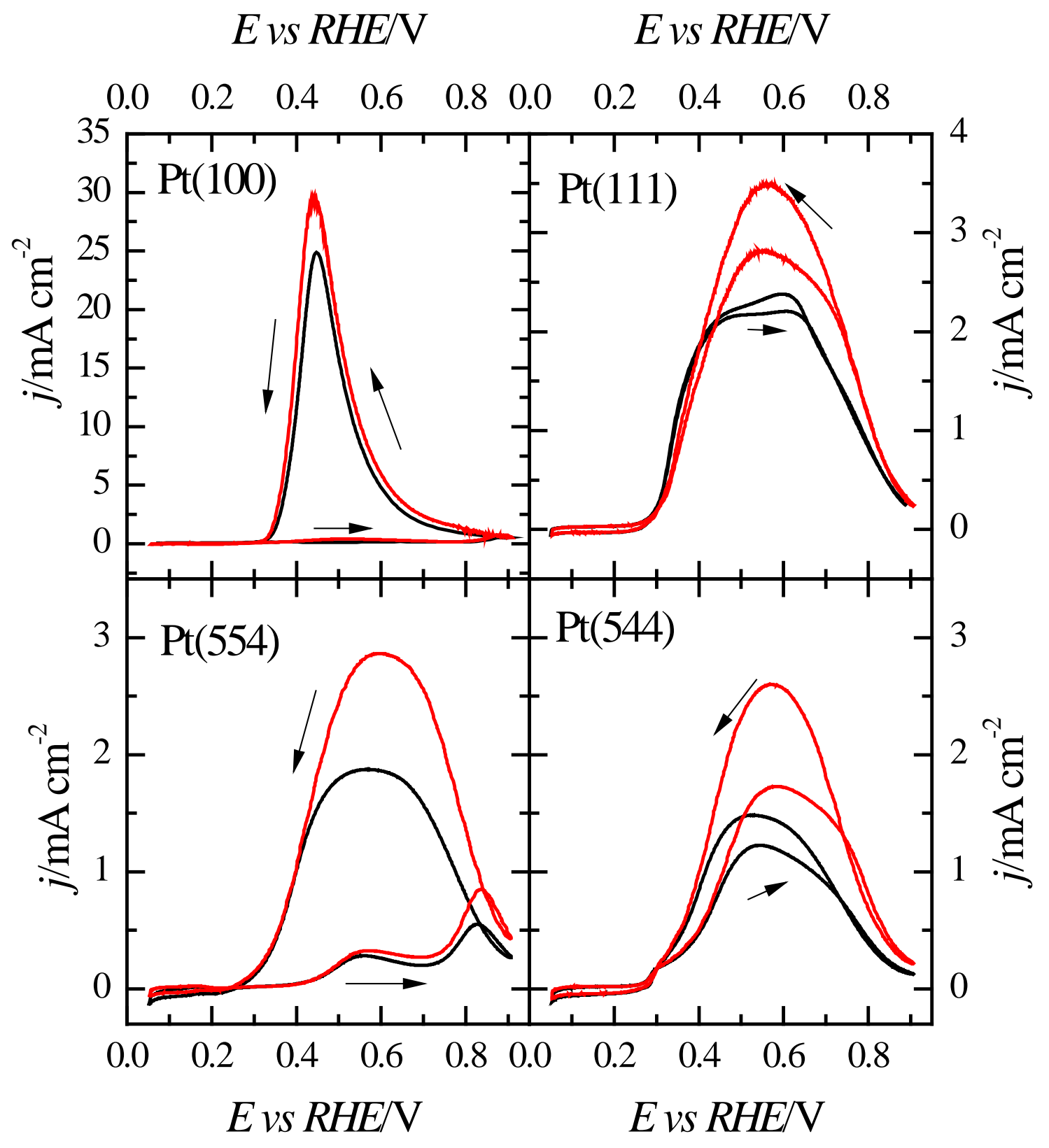

Figure 3. Voltammetric profiles of the electrodes in $0.5 \mathrm{M} \mathrm{H}_{2} \mathrm{SO}_{4}+0.1 \mathrm{M} \mathrm{HCOOH}$

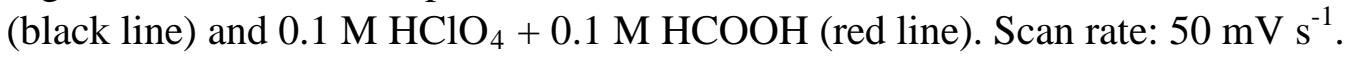




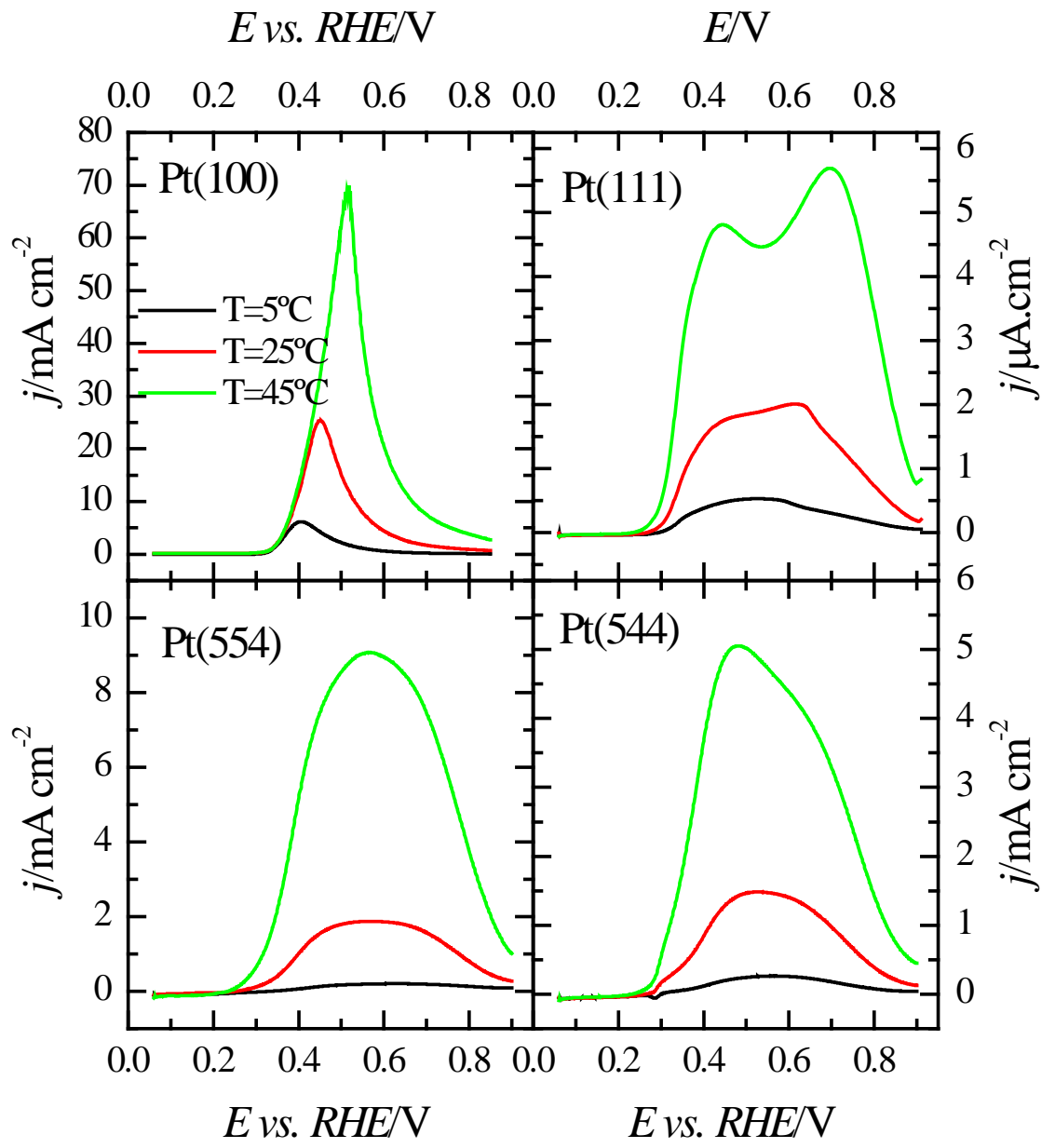

Figure 4. Effect of the temperature in the voltammetric profiles of the electrodes in 0.5 $\mathrm{M} \mathrm{H}_{2} \mathrm{SO}_{4}+0.1 \mathrm{M} \mathrm{HCOOH}$. Only the negative scan direction is shown, for simplicity. Scan rate: $50 \mathrm{mV} \mathrm{s}^{-1}$. 


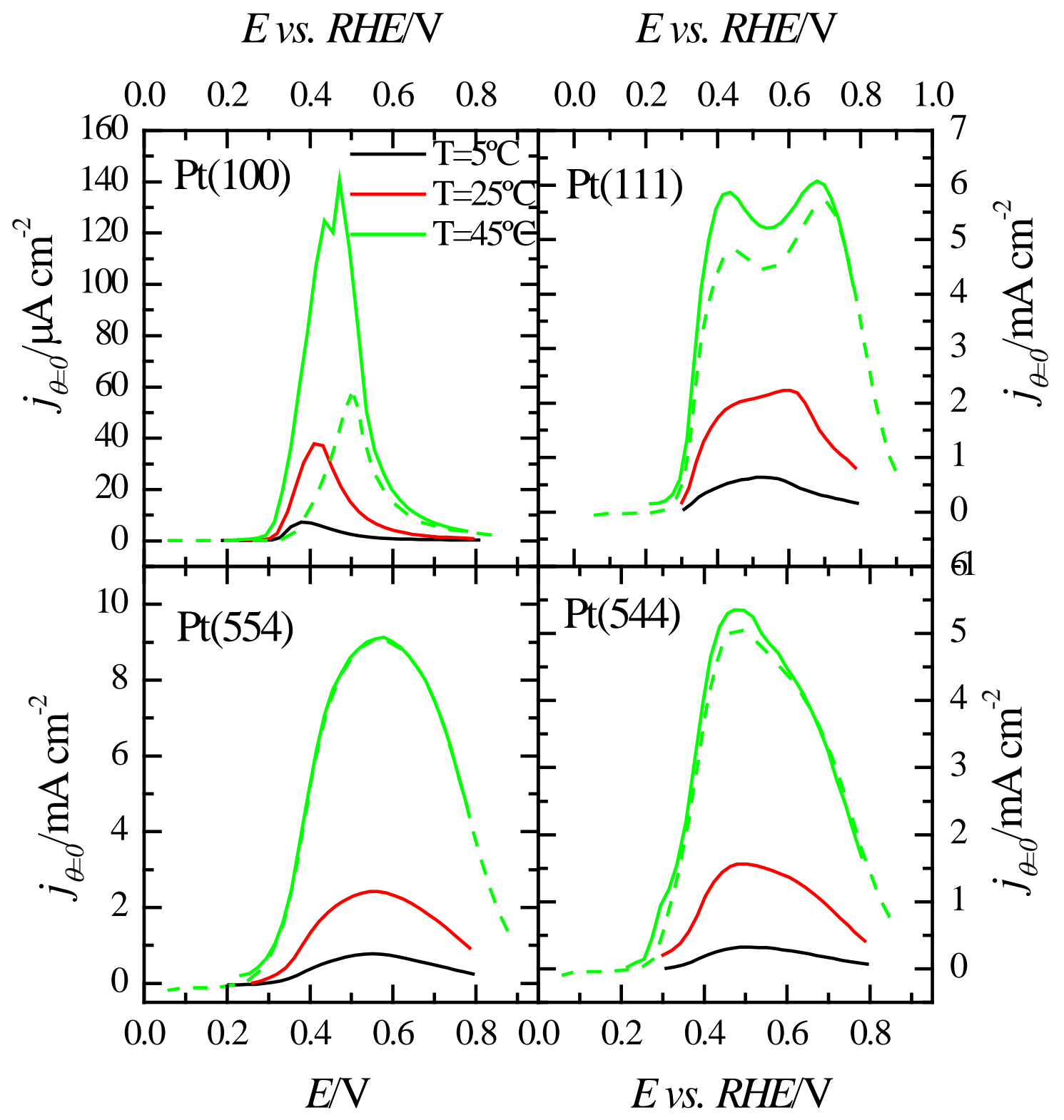

Figure 5. Measured values of $j_{\theta=0}$ at three different temperatures in $0.5 \mathrm{M} \mathrm{H}_{2} \mathrm{SO}_{4}+0.1$ $\mathrm{M} \mathrm{HCOOH}$ for the different electrodes. The negative scan direction at $45{ }^{\circ} \mathrm{C}$ of the voltammetric profile is also shown for comparison. 


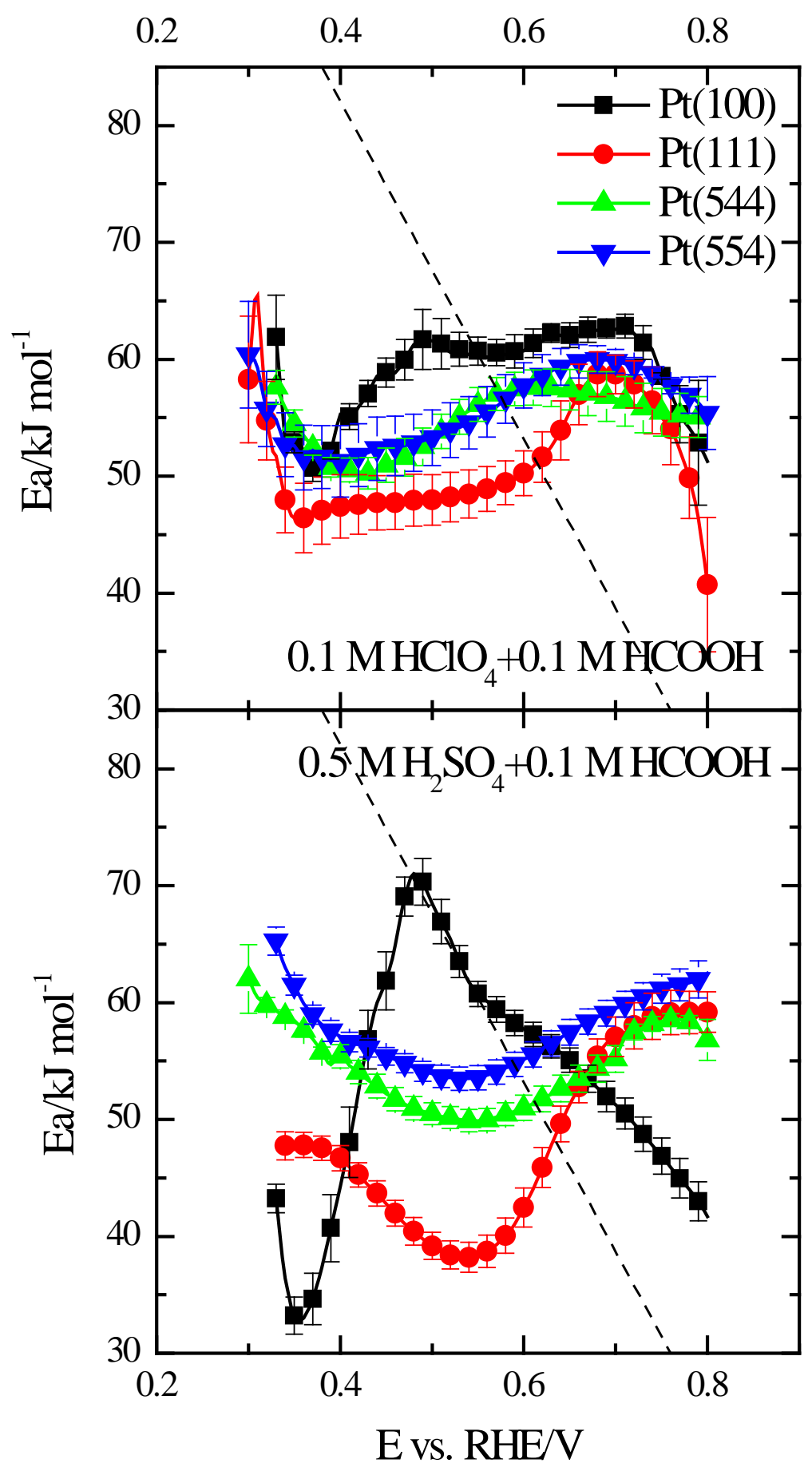

Figure 6. Calculated apparent activation energy for the direct oxidation of formic acid for the different electrodes in $0.5 \mathrm{M} \mathrm{H}_{2} \mathrm{SO}_{4}+0.1 \mathrm{M} \mathrm{HCOOH}$ and $0.1 \mathrm{M} \mathrm{HClO}_{4}+0.1$ $\mathrm{M} \mathrm{HCOOH}$. The dashed line shows the expected behavior from equation (13), for $\alpha=1.5$ and an arbitrary value of $E a^{\neq \text {. }}$ 


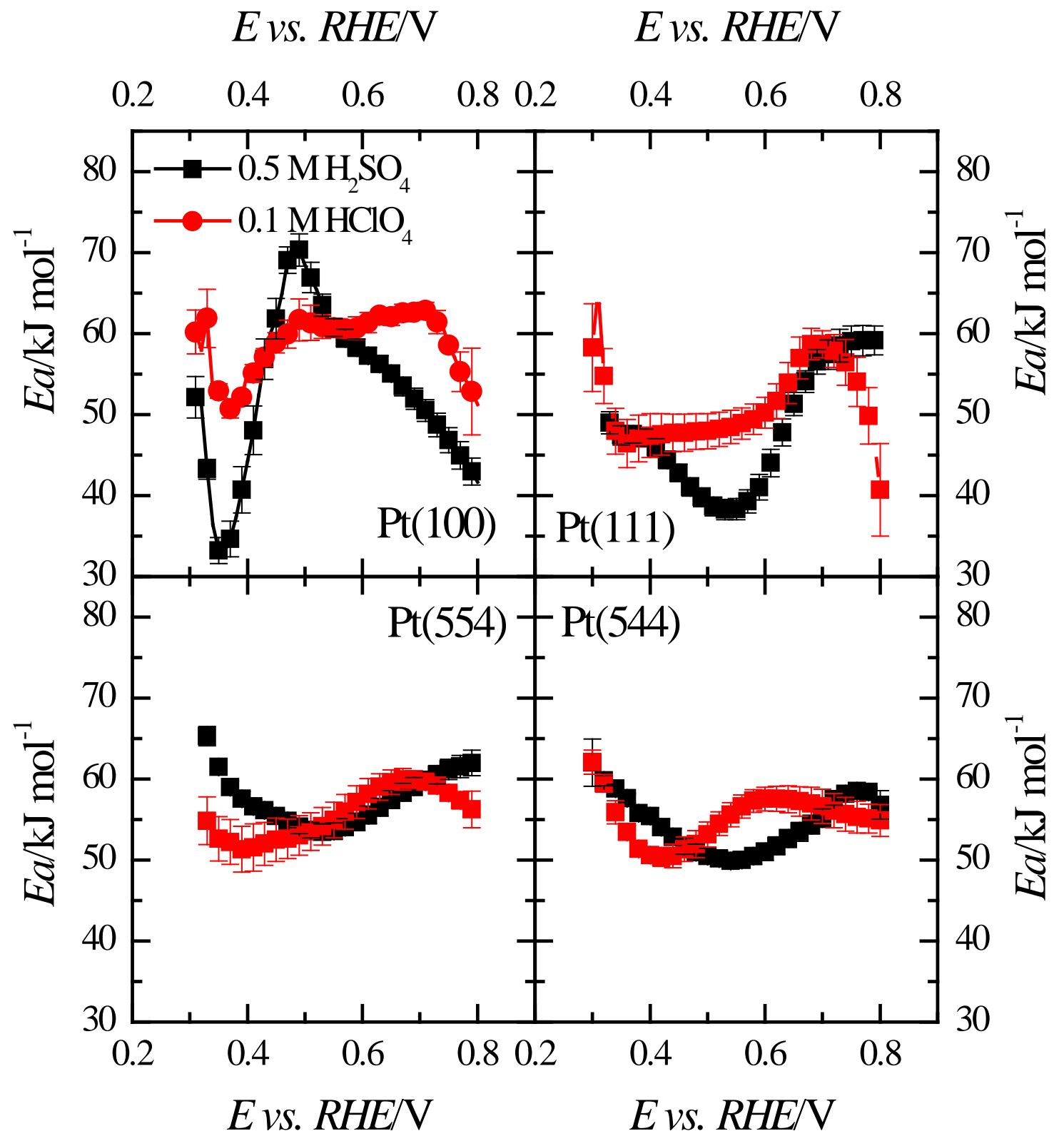

Figure 7. Effect of the electrolyte composition in the apparent activation energy for the direct oxidation of formic acid of the electrodes $0.5 \mathrm{M} \mathrm{H}_{2} \mathrm{SO}_{4}+0.1 \mathrm{M} \mathrm{HCOOH}$ (black curves) and $0.1 \mathrm{M} \mathrm{HClO}_{4}+0.1 \mathrm{M} \mathrm{HCOOH}$ (red curves). 


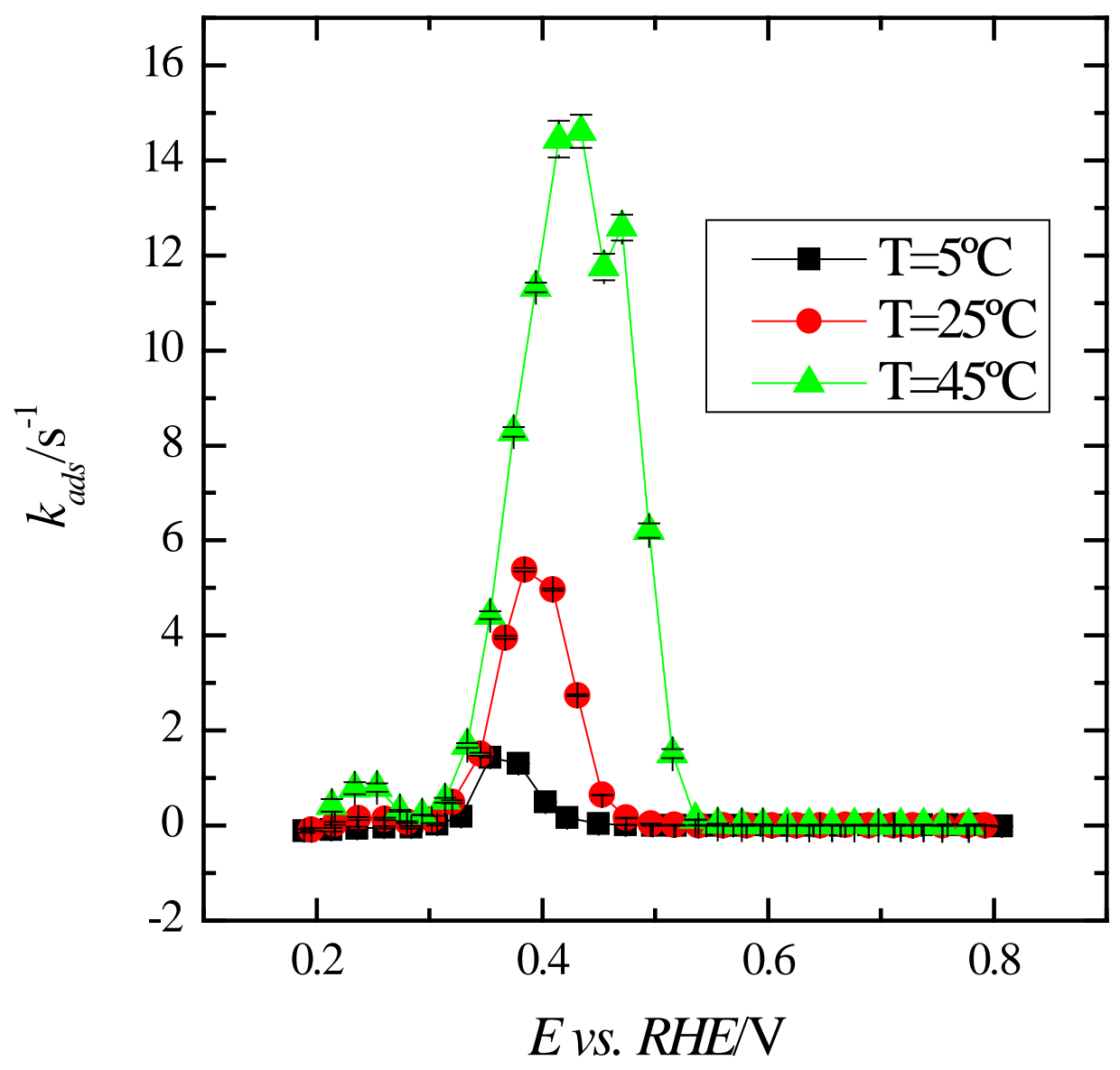

Figure 8. Measured values of $k_{a d s}$ at three different temperatures in $0.5 \mathrm{M} \mathrm{H}_{2} \mathrm{SO}_{4}+0.1$ M HCOOH for the Pt(100) electrode. 


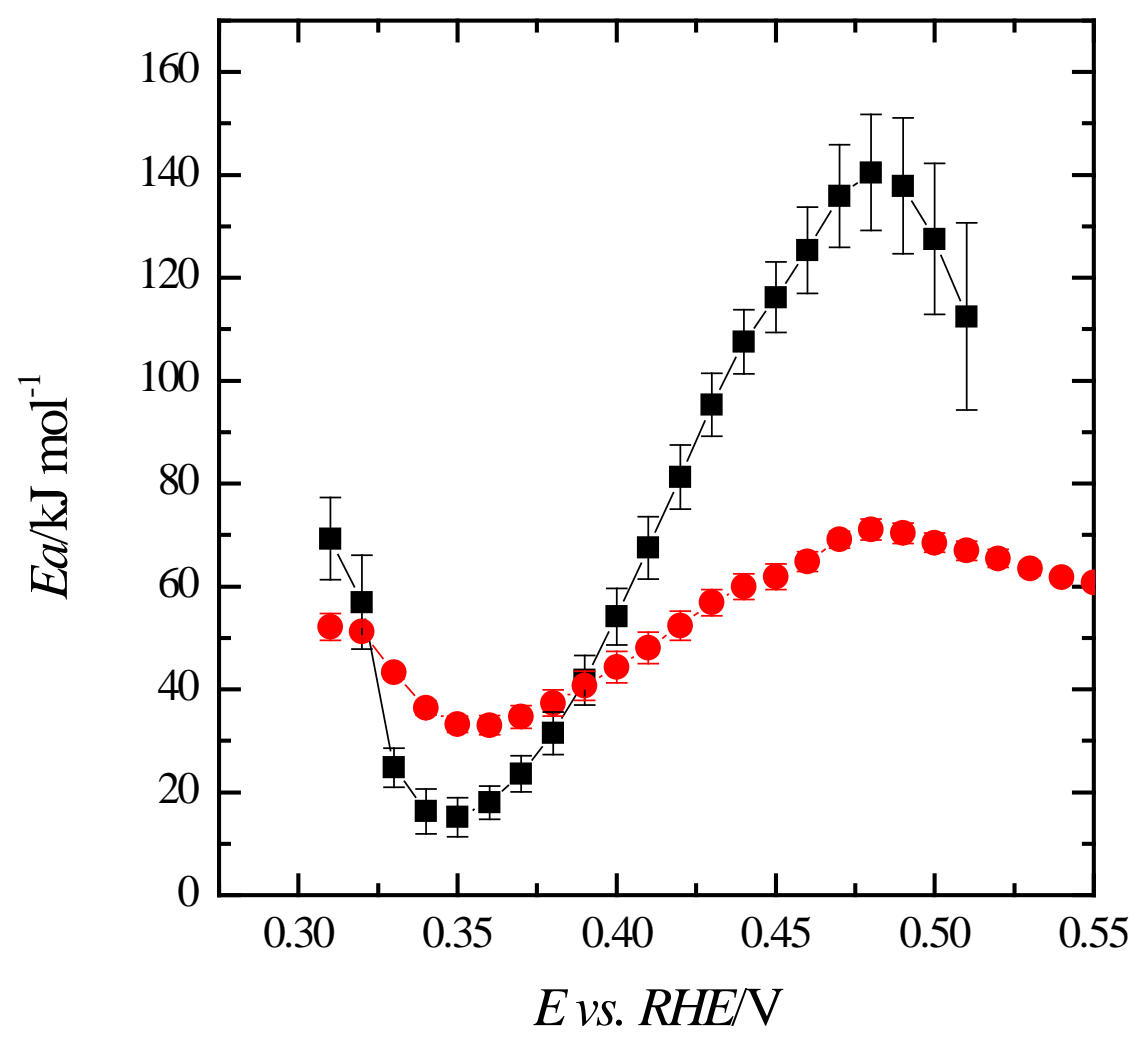

Figure 9. Calculated apparent activation energy for the formation of $\mathrm{CO}$ from formic acid (black points) and the direct oxidation of formic acid (red points) in $0.5 \mathrm{M} \mathrm{H}_{2} \mathrm{SO}_{4}$ $+0.1 \mathrm{M} \mathrm{HCOOH}$ on the Pt(100) electrode. 